\title{
Molecular Characterization of Extended-Spectrum Beta-Lactamase Producing Urinary Escherichia coli Isolated in Brong-Ahafo Regional Hospital, Ghana
}

\author{
Wireko, Solomon, \\ Regional Hospital, Sunyani \\ E. H. Frimpong, \\ P. K. Feglo,
}

Department of Clinical Microbiology, School of Medical Sciences, Kwame Nkrumah University of Science and Technology, Kumasi

\begin{abstract}
Introduction. Antimicrobial resistance is a growing international problem resulting from the enzyme $\beta$-lactamase production by bacteria, to degrade antibiotics, especially $\beta$-lactam antibiotics. In Brong Ahafo Regional Hospital, Sunyani, these antibiotics are heavily depended upon for the treatment of serious infections, but unfortunately high proportions of bacterial isolates in the hospital, have been found to be resistant to the commonly prescribed antibiotics. This study aims to determine the prevalence of ESBL-producing E. coli so as to determine if ESBL are responsible for the high antimicrobial resistance seen at the Brong-Ahafo Regional Hospital, Sunyani.
\end{abstract}

Methods. The study was a cross sectional study, involving $51 \mathrm{E}$. coli isolates from urine samples of both in- and out-patients between January and December, 2014. Antimicrobial susceptibility profiles of the isolates were determined by the Kirby Bauer disc diffusion method against 12 antibiotics. The isolates were screened for ESBL production, and then confirmed by the combined disc method. The isolates were tested for the presence of ESBL blaстХ-м and bla TEM by conventional PCR.

Results. Non-repeat 1,302 midstream urine samples were cultured from which 200 different pathogens were isolated. Of the 200 isolates 51 were E. coli. Isolates obtained from Community isolates were 16(37.2) and isolates from in-patients were 27(62.8). Resistant strains were detected to all the 12 antimicrobials tested. Low proportions were sensitive to cephalosporins (cefotaxime and ceftazidime), both recording 8/51(15.7\%), and quinolones (nalidixic acid and ciprofloxacin), 7/51(13.7\%) and 8/51(15.7\%) respectively. The isolates had varied susceptibility to aminoglycosides, with 
low sensitivity of $1 / 32(2.0 \%)$ to gentamicin, but a high proportion of $47 / 51(92.2 \%)$ was sensitive to amikacin. High proportion of the isolates [43/51(84.3\%)] were ESBL phenotype, and was found to be significantly associated $(\mathrm{p}<0.001)$ with antimicrobial resistance among the isolates. The most prevalent ESBL genotype was Blatem with $26(66.7 \%)$ and blactX-M $28(71.8 \%)$, but $9(23.1 \%)$ ESBL phenotypes tested negative for both blaTEM and blactх-м genes. $22(56.4 \%)$ of the isolates had both blaтем and blacтх-м genes.

Conclusion. High proportions of E. coli isolates from urine in Sunyani produce ESBLs. Both blaтем and blaстХ-м were prevalent and linked to the high levels of drug resistance found in the locality. Increased antibiotic stewardship and stringent infection control measures along with the testing for ESBL must be instituted in the hospital.

Keywords: Extended-Spectrum Beta-lactamase, UTI, antibiotic resistance, CTX-M and TEM.

\section{Introduction}

Overuse of antibiotics is regarded as one of the many reasons for the rising trend of resistance observed among different pathogens, (Gonçalves et al., 2016) all over the world and grave concern has been expressed about the increase in the numbers of organisms that are multi-drug resistant and the difficulty encountered in their treatment (Hima-Lerible et al., 2003; Howladar and Gandhi, 2016). In the choice of antimicrobial for treatment, $\beta$ lactam antimicrobials are preferred because they are highly effective against several bacterial pathogens, with minimal side effects and reduced toxicity, (Chiriac et al., 2016) as a result, $\beta$-lactam antibiotics are frequently prescribed and oftentimes indiscriminately (Newman et al., 2006; Bassetti et al., 2016). As a result, resistance to $\beta$-lactam antibiotics is substantially on the ascendancy becoming a frequent problem encountered in medical practice (Fisher et al., 2005). As the introduction of third generation cephalosporins into any community comes along with it the emergence of ESBLs, (Pitout et al., 2004; Paterson and Bonomo, 2005; Perez et al., 2007). $\beta$-lactamase production effectively terminates $\beta$-lactam activity and serve as the predominant resistance mechanism among Gram-negative bacteria including $E$ coli (Bonnet, 2004; Hertz et al., 2016). ESBLs have extended activity against alternative antibiotic groups like the aminoglycosides and the fluoroquinolones, so ESBL-producing organisms become resistance to them ((Ramazanzadeh, 2010).

In Ghana these antibiotics are the mainstay for the treatment of many infections including UTI, but routine screening of isolates for ESBLs is not done, because of the additional cost it constitutes to the patient, lack of 
testing facilities to the technicians, and the general lack of interest among clinicians (Newman et al., 2006). Heavy dependence of $\beta$-lactam antibiotics leads to the development of $\beta$ - lactamases and resistance as evident in high resistance proportions reported from teaching Hospitals in Ghana mainly Korle Bu Teaching Hospital(KBTH) (Opintan and Newman, 2007; ObengNkrumah et al., 2013 ) and Komfo Anokye Teaching Hospital (KATH) (Feglo et al., 2013). Studies on ESBLs have not been conducted from secondary referral hospitals such as the Brong Ahafo Regional Hospital, in Sunyani. This study therefore is to determine the prevalence of ESBL phenotype and genotypes among $E$. coli strains obtained from UTI patients at Sunyani and to determine their antimicrobial susceptibility patterns.

\section{METHODS}

\section{Study design and setting}

This was a cross-sectional study conducted at the Brong Ahafo Regional Hospital, which is a 300-bed and secondary referral hospital in the Brong Ahafo Region, with population of about two million inhabitants. The Region has 19 districts with Sunyani as the Regional capital.

Urine samples for the study were obtained from patients referred to the Microbiology Laboratory of the hospital for UTI investigations. Patients who gave their informed consent had their samples included in the study. Ethical clearance was obtained from the Institutional Ethics Committee of the Brong- Ahafo Regional Hospital, Sunyani.

\section{Bacterial isolation}

Early morning, mid-stream urine samples were received from patients at the Microbiology laboratory and plated on cysteine lactose electrolyte deficient (CLED) agar using a $1 / 400 \mu \mathrm{m}$ calibrated loop and pure colonies of $\geq 25$ growing on CLED agar after overnight incubation at $37^{\circ} \mathrm{C}$ were considered significant for diagnosis of urinary tract infection. This is equivalent to growth of $10^{5}$ colony forming units/ml of one organism type and was considered significant bacteriuria. Where there were mixed growth of different organisms it was considered contamination and then rejected.

The growths were identified by their colonial growth morphology on CLED medium. Lactose fermenting colonies, suspected to be E. coli were tested further using Gram stain (Gram negative rods), positive in the motility test and then indole and methyl red positivity, citrate negative and VogesProskauer negative tests.

\section{Antibiotic susceptibility testing}

Antibiotic sensitivity of the isolates was determined by the modified Kirby-Bauer method according the CLSI recommended guidelines (20th Inf. 
Suppl. M100-S20). Each set of tests was controlled using susceptible E. coli control strain (ATCC 25922). Antimicrobials and their concentrations tested included: Ampicillin/Sulbactam 20ug, amikacin 30ug, cefotaxime 30ug, ceftazidime 30ug, chloramphenicol 30ug, ciprofloxacin 5ug, gentamicin 10ug, ofloxacin 5ug, nalidixic acid 10ug, tetracycline 30ug, Levofloxacin 5ug, and ceftizoxime 30ug.

\section{ESBL Screening and confirmation}

In the routine susceptibility testing strains with reduced zone diameters of $<27 \mathrm{~mm}$ and $<22 \mathrm{~mm}$ respectively to cefotaxime and or ceftazidime were suspected of producing ESBLs according to CLSI guidelines (CLSI, 2011). All suspect isolates in the screening test were confirmed using the cefpodoxime discs alone and combined discs of cefpodoxime and clavulanic acid $(30 \mu \mathrm{g}+10 \mu \mathrm{g})$ (Oxoid $\mathrm{GmbH}$, Wesel, Germany). Isolates that produced increased zones of $\geq 5 \mathrm{~mm}$ between cefpodoxime disc alone and the combined disc is considered an ESBL. Escherichia coli ATCC 25922 was used as ESBL negative control and Klebsiella pneumoniae ATCC 700603 was used as ESBL positive control.

\section{Molecular Characterization of ESBLs}

All confirmed ESBL phenotypes were tested for Bla $a_{\mathrm{TEM}}$ and $b l a_{\mathrm{CTX}-\mathrm{M}}$ by PCR according previously described techniques (Monstein et al., 2007) and (Mulvey et al., 2003) Mulvey et al., 2004).

Table 1. Primers for amplification of bla $a_{\mathrm{TEM}}$ and $b l a_{\mathrm{CTX}-\mathrm{M}}$

\begin{tabular}{|c|c|c|c|c|}
\hline Gene & & Primer sequence (5"-3") & $\begin{array}{l}\operatorname{siz} \\
\mathrm{e}\end{array}$ & Source \\
\hline \multirow[b]{2}{*}{ Blatem } & TEM-F & ATGTGGCAGYACCAGTAARGTKTGGC & \multirow[b]{2}{*}{593} & \multirow{2}{*}{$\begin{array}{l}\text { Monstein \& } \\
\text { LE, (2007) }\end{array}$} \\
\hline & TEM-R & TGGGTRAARTARGSACCAGAAYCAGCG & & \\
\hline \multirow[t]{2}{*}{ Bla стх-м } & $\begin{array}{l}\text { CTX-M- } \\
\text { U1 }\end{array}$ & ATGTGCAGYACCAGTAARGTKATGGC & \multirow[t]{2}{*}{593} & \multirow[t]{2}{*}{$\begin{array}{l}\text { Mulvey et al. } \\
\text { (2003) }\end{array}$} \\
\hline & $\begin{array}{l}\text { CTX-M- } \\
\text { U2 }\end{array}$ & $\begin{array}{l}\text { TGGGTRAARTARGTSACCAGAAYCAGC } \\
\text { GG }\end{array}$ & & \\
\hline
\end{tabular}

Source: Bla CTX-M (Mulvey et al.,2003), and Bla TEM( Monstein \& LE, 2007) Key for standard Mixed base symbols: R-A,G; Y-C,T; M-A,C; K-G,T; S-C,G; W-A,T; H-A,C,T; BC,G,T; V-A,C,G; D-A,G,T(Integrated DNA Technologies, Inc , USA)

\section{Data Processing and Analysis}

Continuous data were expressed as mean $\pm \mathrm{SD}$ and categorical data expressed as proportion. Resistance proportions were compared using chisquare tests. In all cases a p-value $<0.05$ was considered significant. The data were analyzed using Stata/IC 10.0 for windows (StataCorp LP, USA, http://www.stata.com). 


\section{RESULTS}

\section{Antibiotic susceptibility pattern of $E$. coli}

Non-repeat midstream urine samples numbering 1,302 were cultured. Significant bacteriuria was detected and 200 different pathogens were isolated. Of the 200 isolates 51 were E. coli. E. coli isolates obtained from community isolates were 16(37.2) and 27(62.8) were obtained from inpatients. All the 51 E. coli isolates were tested against 12 different antibiotics namely Ampicillin/Sulbactam, nalidixic acid, cefotaxime, ceftazidime, chloramphinicol ciprofloxacin, ceftizoxime, tetracycline, ofloxacin, gentamicin, amikacin and levofloxacin, using the Disk-diffusion method.

High proportions of the $E$. coli isolates were resistant to the various antimicrobial agents tested. The highest resistant proportions were found against tetracycline and ampicillin where they recorded (50/51 (98.0\%) and $(38 / 51(74.5 \%)$ respectively. Varied resistant proportions were detected to the quinolones with 43/51(84.3\%) to nalidixic acid and ofloxacin recorded 36/51(70.6\%), levofloxacin 27/51(52.9\%) and ciprofloxacin 42/51(82.4\%). Resistant proportions were detected against aminoglycosides and cephalosporins tested and the details are presented in table 2 .

Table 2. Antibiotic susceptibility pattern of E. coli isolated from urine at Sunyani.

\begin{tabular}{lcccccc}
\hline $\boldsymbol{E}$. coli $(\boldsymbol{n}=\mathbf{5 1})$ & $\mathbf{A S}$ & $\mathbf{N X}$ & $\mathbf{C T X}$ & $\mathbf{C A Z}$ & $\mathbf{C H}$ & $\mathbf{C P}$ \\
\hline Sensitive & $5(9.8 \%)$ & $7(13.7 \%)$ & $8(15.7 \%)$ & $8(15.7 \%)$ & $16(31.4 \%)$ & $8(15.7 \%)$ \\
Resistant & $38(74.5 \%)$ & $43(84.3 \%)$ & $42(82.4 \%)$ & $42(82.4 \%)$ & $34(66.7 \%)$ & $42(82.4 \%)$ \\
& & & & & & \\
Intermediate & $8(15.7 \%)$ & $1(2.0 \%)$ & $1(2.0 \%)$ & $1(2.0 \%)$ & $1(2.0 \%)$ & $1(2.0 \%)$ \\
Total & $\mathbf{5 1}$ & $\mathbf{5 1}$ & $\mathbf{5 1}$ & $\mathbf{5 1}$ & $\mathbf{5 1}$ & $\mathbf{5 1}$ \\
\hline
\end{tabular}

\begin{tabular}{lcccccc}
\hline $\boldsymbol{E} . \boldsymbol{c o l i}(\boldsymbol{n}=\mathbf{5 1})$ & $\mathbf{C L}$ & $\mathbf{T E}$ & $\mathbf{O F}$ & $\mathbf{G M}$ & $\mathbf{A K}$ & $\mathbf{L E}$ \\
\hline Sensitive & $31(60.8 \%)$ & - & $11(21.6 \%)$ & $1(2.0 \%)$ & $47(92.2 \%)$ & $11(21.6 \%)$ \\
Resistant & $12(23.5 \%)$ & $50(98.0 \%)$ & $36(70.6 \%)$ & $30(58.8 \%)$ & $2(3.92 \%)$ & $27(52.9 \%)$ \\
& $8(15.7 \%)$ & $1(2.0 \%)$ & $4(7.8 \%)$ & $1(2.0 \%)$ & $2(3.9 \%)$ & $13(25.5 \%)$ \\
$\begin{array}{l}\text { Intermediate } \\
\text { Total }\end{array}$ & $\mathbf{5 1}$ & $\mathbf{5 1}$ & $\mathbf{5 1}$ & $\mathbf{3 2}$ & $\mathbf{5 1}$ & $\mathbf{5 1}$ \\
\hline
\end{tabular}

AS: Ampicillin/Sulbactam; NX: Nalidixic acid; CTX: Cefotaxime; CAZ: Ceftazidime; CL:

Ceftriaxone; CH: Chloramphenicol; CP: Ciprofloxacin; TE: Tetracycline; OF: Ofloxacin;

GM: Gentamicin; AK: Amikacin; LE: Levofloxacin.

\section{Prevalence of ESBL in urinary E. coli isolated from Sunyani}

Aminoglycoside resistance was also significantly associated with ESBL expression $(\mathrm{p}<0.0006)$. Twenty-seven strains $(90.0 \%)$ were resistant to at least 1 of the 2 tested aminoglycosides with most frequently observed resistance against gentamicin $27(90.0 \%)$, while about $15(35 \%)$ were resistant to quinolones. Of the $51 \mathrm{E}$. coli isolates, 43 tested positive and 8 tested 
negative for ESBL production. There was no significant association between ESBL production, gender and age $(\mathrm{p}<0.42)$ and $(\mathrm{p}<0.18)$ respectively (Table $3)$.

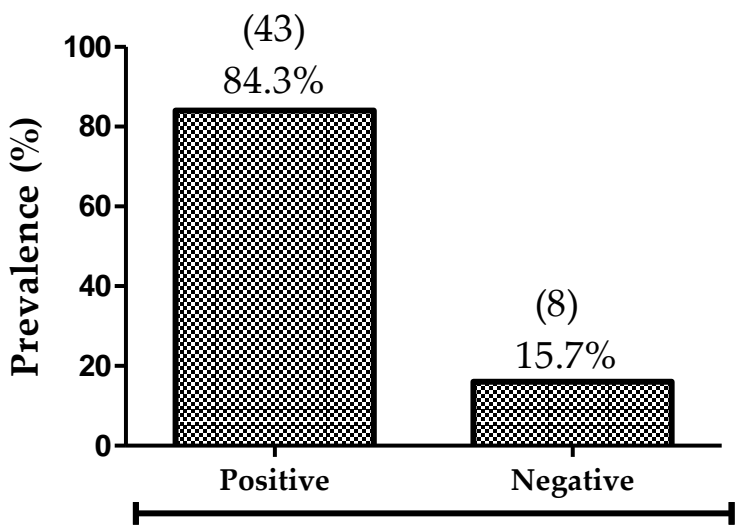

EBLS producing E. coli

Fig 1 Prevalence of EBL producing E. coli isolated from Sunyani

Table 3. Association of Gender and Age with ESBL production in E. coli

\begin{tabular}{lllll}
\hline & Total & \multicolumn{2}{c}{ ESBL } & \\
& $\mathrm{n}=51$ & Positive $\mathrm{n}=43$ & Negative $\mathrm{n}=8$ & p-value \\
\hline Gender & 18 & $14(32.6 \%)$ & $4(50.0 \%)$ & 0.4297 \\
Male & 33 & $29(67.4 \%)$ & $4(50.0 \%)$ & - \\
Female & & & & \\
Age group (years) & 9 & $8(18.6 \%)$ & $1(12.5 \%)$ & 0.1828 \\
Under five years & 42 & $35(81.4 \%)$ & $7(75.0 \%)$ & - \\
Adults & & & & \\
\hline
\end{tabular}

Values are presented as frequency (percentage). Difference between proportions was tested using Chi-square. $\mathrm{p}<0.05$ was considered statistically significant.

There was significant association between ESBL production and multi-drug resistance. Antimicrobial resistance was significantly associated with ESBL production $(\mathrm{p}<0.0001)$ (Table 4).

Table 4 Comparison between antimicrobial drug resistance and ESBL production in E. coli

\begin{tabular}{llll}
\hline & $\begin{array}{l}\text { ESBL isolates } \\
\mathbf{N}=\mathbf{4 3}\end{array}$ & $\begin{array}{l}\text { Non-ESBL } \\
\text { isolates } \\
\mathbf{N = 8}\end{array}$ & Total (N=51) \\
\hline & $\%$ & $\begin{array}{l}\% \\
\text { Resistance }\end{array}$ & P-values \\
\hline Resistance & $0 / 42(0.0)$ & $<\mathbf{0 . 0 1}$ \\
\hline Ceftazidime & $42 / 42(100.0)$ & $0 / 42(0.0)$ & $<\mathbf{0 . 0 1}$ \\
\hline
\end{tabular}




\begin{tabular}{lllc}
\hline Ceftizoxime & $9 / 12(75.0)$ & $3 / 12(25.0)$ & $<\mathbf{0 . 0 1}$ \\
\hline Ciprofloxacin & $36 / 42(85.7)$ & $6 / 42(14.3)$ & 0.84 \\
\hline Levofloxacin & $24 / 27(88.9)$ & $3 / 27(11.1)$ & $<\mathbf{0 . 0 1}$ \\
\hline Ofloxacin & $32 / 36(88.9)$ & $4 / 36(11.1)$ & $<\mathbf{0 . 0 1}$ \\
\hline Nalidixic acid & $37 / 43(86.0)$ & $6 / 43(14.0)$ & 0.87 \\
\hline Amikacin & $2 / 2(100)$ & $0 / 2(0.0)$ & $<\mathbf{0 . 0 1}$ \\
\hline Gentamicin & $27 / 30(90.0)$ & $3 / 30(10.0)$ & $<\mathbf{0 . 0 1}$ \\
\hline $\begin{array}{l}\text { Ampicillin- } \\
\text { sulbactam }\end{array}$ & $33 / 38(86.8)$ & $5 / 38(15.2)$ & $<\mathbf{0 . 0 1}$ \\
\hline Tetracycline & $42 / 50(82.4)$ & & $<\mathbf{0 . 0 1}$ \\
\hline
\end{tabular}

Continuous data were presented as mean \pm Sd and categorical data presented as proportion. Categorical data were compared to each other using Chi-square analysis. 0.05

\section{Molecular Characterization of ESBLs}

The two ESBL genes namely Bla detected among the 43 E. coli ESBL phenotypes. Bla (66.7\%). Bla being $51.1 \%$ of the isolates were positive for both Bla CTX-M and Bla genes. Phenotypically $9(20.9 \%)$ of isolates tested positive for ESBL but were negative for both $B l a_{\mathrm{CTX}-\mathrm{M}}$ and $B l a_{\mathrm{TEM}}$. Pictures of agarose gel separation of amplicons for $B l a_{\text {TEM }}$ and $B l a_{\text {CTX-M }}$ are shown in Figures 2 and 3.

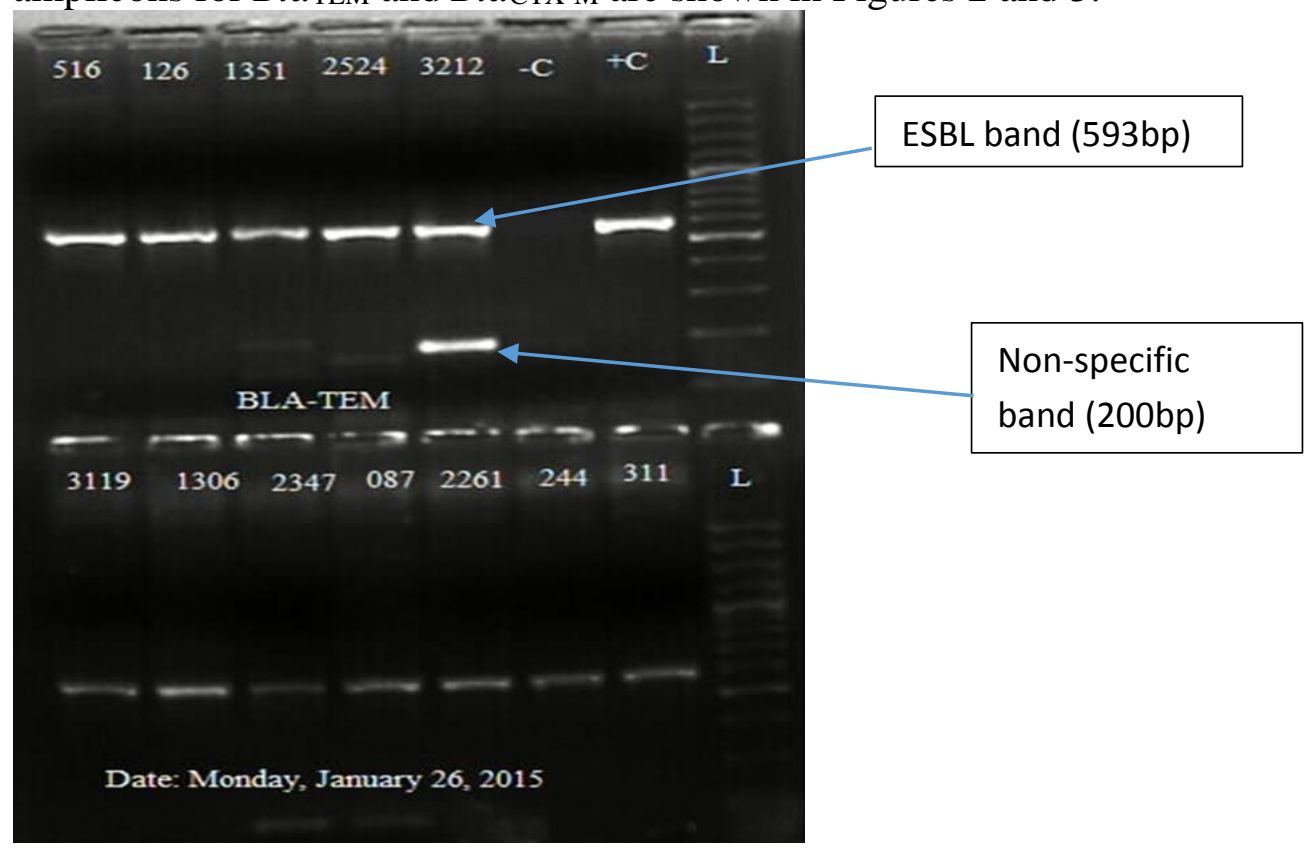

Isolates 087, 3212 and 2347 showed non- specific bands (presence of two genes) of 593/200 while 876, 947, 2183, 2908, 4173 and 314 are negative for blaTEM $+c$ depicts $P C R$ product of E. coli positive control and -c depicts PCR product of a negative control.

$L$ is the molecular weight standard 100bp (Thermo Scientific). Band size 593bps

Fig 2. PCR amplicons on $2 \%$ agarose gel for BlaTEM genes of E. coli 


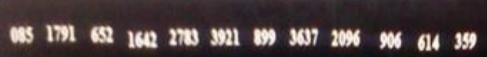

$f+$

Bla CTX-M bands (593pbs)

Isolates 3119**negative for bla CTX-M, $+c$ and $-c$ are positive and negative controls, $L$ is ladder standard 100bp

Fig 3. PCR amplicons on $2 \%$ agarose gel for bla стХ-м genes

\section{DISCUSSIONS}

Several studies have demonstrated the presence of $\beta$-lactamases in Ghana, but most of these studies were conducted in tertiary referral institutions. Results similar to those obtained in tertiary referral hospitals were expected in Sunyani Hospital, because it is a secondary referral hospital and specialist services exist in this institutions too, just like other regional hospitals in Ghana. Patients are referred from many districts within the region and beyond to seek health care at the regional hospitals. It is cases beyond the competence of the regional hospitals that are referred to the tertiary levels in Kumasi and Accra. This problem of ESBL has not caught the attention of the health authorities and are missing in many medical reports from the health institutions. ESBLs are not just nosocomial infections in big hospitals in Ghana, but are spreading into the communities as well (Feglo et al., 2013). In this present study 43/51(84\%) of the urinary E. coli isolates produced ESBL and 16(37.2\%) were community isolates, an observation, which confirms findings in other studies that ESBL-producing Enterobacteria are detectable in both community and hospitalized patients with varying prevalence levels as $63 \%$ was reported from Mali (Tande et al., 2009), 43\% in Ghana (Feglo et al., 2013), 40\% in Niger (Woerther et al., 2011 ) and $26 \%$ in Kenya (Kariuki et al., 2007) and $28.66 \%$ in Bangladesh (Islam et al., 2017). It was estimated that ESBL prevalence of $80 \%$ in a hospital setting will lead to as much as $80 \%$ of treatments failures and 35\% 
mortality (Perez et al., 2007). The detection of more than $80 \%$ ESBL prevalence in Sunyani is of concern as deaths might have arisen from treatment failures as a result of ESBL problem in the Sunyani health facility. There are no ESBL records and ESBL treatment failures are not and cannot be accounted for because the hospital authorities are unaware of it and $\beta$ lactam antibiotics are still prescribed. Even if hospital ESBL spread is controlled, the community ESBL auxotrough spread will persist and will replace normal antibiotic susceptible wild type in the community. Alternative antibiotic groups like the aminoglycosides and the fluoroquinolones, have also been affected by the ESBL menace because the enzymes have extended activity to non-beta lactam antibiotics too (Ramazanzadeh, 2010). As demonstrated in the present study, appreciable proportion of $E$. coli resistance was recorded against the quinolones, with resistance proportion to nalidixic acid being $84.3 \%$, ofloxacin $(70.6 \%)$, Levofloxacin (52.9\%) and ciprofloxacin (82.4\%).

ESBL carriage was observed to be significantly associated with antimicrobial resistance in $E$. coli isolates tested from Sunyani ( $<<0.01$ ), as was also observed in India (Jain et al., 2003) Bangladesh (Asna et al., 2017). Studies indicate that patients who have ESBL infection seem to have poorer prognosis than patients infected with non ESBL bacteria (Paterson and Bonomo, 2005), and the reason may be due to treatment failures. This is a serious issue given that immunity at extremes of life is relatively weak, a situation which prescribers must know of and must show optimum care for in the choice of antibiotics meant for patients less than 5years and above 60 years.

There was increased resistance of $E$. coli to most of the antimicrobials tested, with the exception of amikacin to which resistant proportion was low (3.9\%). Though $20.26 \%$ of community urinary isolates in Morocco were found to be multidrug resistant (Natoubi et al., 2017), elsewhere in China and India, amikacin and nitrofurantoin were reported to be effective drugs for the treatment for UTIs (Shao et al., (2004) and Mandira Mukherjee et al., (2013) respectively. In our study and in Nigeria high proportions of urinary isolates were resistant with all $E$. coli isolates $(100 \%)$ being resistant to augmentin, gentamycin, streptomycin, tetracyclin and chloramphenicol, and $90.90 \%$ resistant to ofloxacin, sparfloxacin and amoxicillin (Elikwu et al., 2017). High resistant proportions seen in this current study is attributable to many factors. Firstly, many hospitals in Ghana do not have the competence and the facility to do bacterial culture, isolation and antimicrobial sensitivity testing, so physicians prescribe without laboratory support. The second factor is the indiscriminate use the drugs, because they are relatively cheap and easily accessible by the patient. The drugs can be purchased off the counter due to lack of enforcement of 
regulations, and also because some of these antibiotics are taken orally, so they are easy to administer and misuse (Newman et al., 2006). Another link to antibiotic resistance is the increased use of antibiotics in animal husbandry coupled with the production and sale of substandard and fake drugs (Tajick, 2006; Shakoor et al., 1977). In the case of substandard drugs physicians prescribe adequate doses, but do not achieve optimal clinical response invivo leading to the creation of resistant strains.

In this study, Bla $a_{\text {TEM }}$ was detected in $26(66.7 \%)$ of the ESBL E. coli strains and bla CтХ-м tested was detected in 28 (71.8\%) of the isolates. These results are similar to other results obtained in studies in tertiary institutions in Ghana where higher Bla $a_{\text {TEM }}$ were reported than the Bla $a_{\text {CTX-M }}$ among E. coli strains (Obeng-Nkrumah et al., 2013 ; Labi et al., 2016).

Studies in Europe and America reported more prevalence of SHV and CTX-M- $\beta$-lactamase organisms than the TEM (Bonnet, 2004; Naseer and Sundsfjord, 2011), but most studies in Ghana report the TEM ESBL as the most common, just as it is found in the current study in Sunyani. Though tested phenotypically to be ESBLs about $20.9 \%$ of the ESBL-producing isolates in this study were negative for the both Bla $a_{\mathrm{CTX}-\mathrm{M}}$ and Bla indication that other ESBLs types could be present.

The increased diversity and complexity of $\beta$-lactam resistance in gramnegative organisms, have necessitated the call for stringent infection control and antimicrobial stewardship. Also increased establishment of institutional testing facilities, training of personnel and continuous monitoring of drug resistance Sunyani Hospital is suggested. Testing for other Bla $a_{\text {GENES }}$ such as for SHV, AmpC, OXA, KPC and IMP and VIM in E coli and other members of the Enterobacteriaceae is advocated.

Authors' contribution: EHF conceptualized, designed, supervised and financed the study. WS, did sample collection, laboratory generation of data, and produced the first draft report. PKF was responsible for data analysis and preparation of the manuscript for publication. All authors read and approved the final manuscript.

Competing interests: The authors declare that they have no competing interests.

Consent for publication: Not applicable.

Ethics approval: The study was approved by the Joint Committee on Human Research, Publication and Ethics of School of Medical Sciences and Komfo Anokye Teaching Hospital, Ghana. 
Acknowledgements: The researchers are grateful to Technical Staff at Kumasi Collaborative Centre in tropical medicine (KCCR) for allowing their laboratory space for PCR work.

\section{References:}

1. Asna S.M.Z.H., Akhter S., Rahman M.M., Mohammad N. and Hafez M. (2017) ESBL Positive Organisms: Method of Routine Reporting and Prevalence in Health Care Settings. Bangladesh Journal of Medical Microbiology 8, 23-27.

2. Bassetti M., Welte T. and Wunderink R.G. (2016) Treatment of Gram-negative pneumonia in the critical care setting: is the betalactam antibiotic backbone broken beyond repair? Critical Care 20, 1.

3. Bonnet R. (2004) Growing group of extended-spectrum b-lactamases: the CTX-M enzymes Antimicrob Agents Chemother 48, 1-14.

4. Chiriac A.M., Rerkpattanapipat T., Bousquet P.J., Molinari N. and Demoly P. (2016) Optimal step doses for drug provocation tests to prove beta-lactam hypersensitivity. Allergy.

5. Elikwu C., Shobowale E., Oluyemi O., Afolabi D., Aderinto D., Onyedibe K. and Solarin A. (2017) The etiology and antimicrobial susceptibility patterns of urinary tract infections at a private Nigerian teaching hospital in South West Nigeria. African Journal of Clinical and Experimental Microbiology 18, 21-28.

6. Feglo P., Adu-Sarkodie Y., Ayisi L., Jain R., Spurbeck R.R. and Springman A.C. (2013) Emergence of a novel extended-spectrum beta- lactamase (ESBL)-producing, fluoroquinolone-resistant clone of extra intestinal pathogenic Escherichia coli in Kumasi, Ghana. $J$ Clin Microbiol 51, 728-730.

7. Fisher J.F., Meroueh S.O. and Mobashery S. (2005) Bacterial resistance to $\beta$-lactam antibiotics: compelling opportunism, compelling opportunity. Chemother Rev 105, 395-424.

8. Gonçalves L.F., de Oliveira Martins-Júnior P., de Melo A.B.F., da Silva R.C.R.M., de Paulo Martins V., Pitondo-Silva A. and de Campos T.A. (2016) Multidrug resistance dissemination by extended-spectrum $\beta$-lactamase-producing Escherichia coli causing community-acquired urinary tract infection in the Central-Western Region, Brazil. Journal of Global Antimicrobial Resistance 6, 1-4.

9. Hertz F.B., Schønning K., Rasmussen S.C., Littauer P., Knudsen J.D., Løbner-Olesen A. and Frimodt-Møller N. (2016) Epidemiological factors associated with ESBL-and non ESBLproducing E. coli causing urinary tract infection in general practice. Infectious Diseases 48, 241-245. 
10. Hima-Lerible H., Ménard D. and Talarmin A. (2003) Antimicrobial resistance among uropathogens that cause community-acquired urinary tract infections in Bangui, Central African Republic. $J$ Antimicrob Chemother, 192-194.

11. Howladar A.A. and Gandhi P. (2016) A Study To Determine Prevalence of Quinolone Resistance Genes Among ExtendedSpectrum B-Lactamase-Producing Escherichia Coli. Global Journal For Research Analysis 5.

12. Islam T.A.B., Shamsuzzaman S. and Farzana A. (2017) Prevalence and antibiogram of ESBL producing gram negative bacilli isolated from urine in Dhaka Medical College Hospital, Bangladesh. Bangladesh Journal of Medical Microbiology 9, 17-21.

13. Jain A., Roy I., Gupta M.K., Kumar M. and Agarwal S.K. (2003) Prevalence of extended-spectrum beta-lactamase-producing Gramnegative bacteria in septicaemic neonates in a tertiary care hospital. $J$ Med Microbiol 52, 421-425.

14. Kariuki S., Revathi G., Corkill J., Kiiru J., Mwituria J. and Mirza N. (2007) Escherichia coli from community-acquired urinary tract infections resistant to fluoroquinolones and extended-spectrum betalactams. J Infect Dev Ctries 1 257-262.

15. Labi A.-K., Obeng-Nkrumah N., Bjerrum S., Enweronu-Laryea C. and Newman M.J. (2016) Neonatal bloodstream infections in a Ghanaian Tertiary Hospital: Are the current antibiotic recommendations adequate? BMC Infectious Diseases 16, 598.

16. Monstein H.J., A O.-t.-B., M.V N., M N. and Lee D.K.N. (2007) Multiplex PCR amplification assay for the detection of blaSHV, blaTEM and blaCTX-M genes in Enterobacteriaceae. . APMIS 115, 1400-1408.

17. Mulvey M.R., Soule G., Boyd D., Demezuk W. and Ahmed R. (2003) Characterization of the first Extended Spectrum Beta Lactamase producing Salmonella isolate identified in Canada. . J Clin Microb, 5, 460-462.

18. Naseer U. and Sundsfjord A. (2011) The CTX-M conundrum: dissemination of plasmids and Escherichia coli clones. . Microb Drug Resist 17, 83-97.

19. Natoubi S., Barguigua A., Baghdad N., Nayme K., Timinouni M., Hilali A., Amghar S. and Zerouali K. (2017) Occurrence of Carbapenemases And Extended-Spectrum Beta-Lactamases in Uropathogenic Enterobacteriaceae Isolated From A Community Setting, Settat, Morocco. Asian J Pharm Clin Res 10, 1-5.

20. Newman M.J., Frimpong E., Asamoah-Adu A. and Sampene-Donkor E. (2006) Resistance to antimicrobial drugs in Ghana. The Ghanaian- 
Dutch Collaboration for Health research and Development. Project number 2001/GD/07. Technical report series 5, 8-26.

21. Obeng-Nkrumah N., Twum-Danso K., Krogfelt K.A. and Newman M.J. (2013 ) Extended-Spectrum Beta-Lactamases in Ghana. Am. J Trop Med Hyg 89, 960-964.

22. Opintan J.A. and Newman M.J. (2007) Distribution of serogroups and serotypes of multiple drug resistant Shigella isolates. Ghana Med J. 41, 50-54.

23. Paterson D.L. and Bonomo R.A. (2005) Extended-spectrum betalactamases: A clinical update. Clin Microb Rev 2005, 657-686.

24. Perez F., Endimiani A., Hujer K.M. and Bonomo R.A. (2007) The continuing challenge of ESBLs. Curr Opin Pharmacol 7, 459-469.

25. Pitout J.D., Hanson N.D. and Church D.L. (2004) Population-based laboratory surveillance for Escherichia coli-producing extendedspectrum $\beta$-lactamases: importance of community isolates with bla CTX-M genes. . Clin Infect Dis 38, 1736-1741.

26. Ramazanzadeh R. (2010) Etiologic agents and extended-spectrum beta-lactamase production in urinary tract infections in Sanandaj Iran. Eastern J. Med. 15, 57-62.

27. Tande D., Jallot N., Bougoudogo F., Montagnon T., Gouriou S. and Sizun J. (2009) Extendedspectrum beta-lactamase-producing Enterobacteriaceae in a Malian orphanage Emerg Infect Dis 15, 472474.

28. Woerther P.L., Angebault C., Jacquier H., Hugede H.C., Janssens A.C. and Sayadi S. (2011) Massive increase, spread, and exchange of extended spectrum beta-lactamase-encoding genes among intestinal Enterobacteriaceae in hospitalized children with severe acute malnutrition in Niger Clin Infect Dis 53, 677-685. 